Research Article

\title{
Optimization of Equivalent Source Configuration for an Independent-Equivalent Source Method in Half-Space Sound Field
}

\author{
Wen-Qian Jing $(\mathbb{D}$, Huawei Wu $(\mathbb{D}$, and Jin-Quan Nie \\ Hubei Key Laboratory of Power System Design and Test for Electrical Vehicle, Hubei University of Arts and Science, \\ 296 Longzhong Road, Xiangyang 441053, China
}

Correspondence should be addressed to Huawei Wu; whw_xy@163.com

Received 19 September 2019; Revised 8 November 2019; Accepted 27 November 2019; Published 11 March 2020

Academic Editor: Francesco Franco

Copyright $(2020$ Wen-Qian Jing et al. This is an open access article distributed under the Creative Commons Attribution License, which permits unrestricted use, distribution, and reproduction in any medium, provided the original work is properly cited.

In the situation that vibrating objects are located above a reflecting plane, an independent-equivalent source method (I-ESM) regards the reflections due to the plane as being radiated by equivalent sources placed under the plane and then the half-space sound field is reconstructed by matching the measured pressure with the equivalent sources distributed within the vibrating object and those substituting for reflections. But, this method heavily depends on the equivalent source configuration and may obtain bad reconstruction results if the equivalent sources are arranged incorrectly. This paper deals with the optimization of the equivalent source configuration to ensure I-ESM always perform well. Through numerical simulations and experiments, the influence of equivalent source configurations on the reconstruction accuracy was studied and optimal configurations were acquired and confirmed.

\section{Introduction}

In practice, most vibrating objects are mounted on or located above a plane, resulting in a half-space sound field, and then the reflections from that plane should be considered when using the nearfield acoustic holography (NAH) [1-5] to realize the sound field reconstruction.

By combining with the conventional NAH, the field separation techniques [6-8] can be used to reconstruct the half-space sound field. Also, these techniques can work in the situation that the surface impedance of the reflecting plane is unknown, but they require that the measurement surfaces should surround the vibrating object at a close range. In addition, the field separation techniques require both the pressure and particle velocity measurements if the measurements are made on one single-layer surface [6], or require double-layer measurements if only pressure [7] or particle velocity [8] is measured. As a result, high measurement costs would be needed.

On the condition that the surface impedance is known $a$ prior, half-space Green's function can be introduced into the conventional NAH [9-13] to account for the reflections. Both in $[10,11]$, the reflections were assumed to be as plane waves, which might not obtain a good result if the vibrating object was not far away from the reflecting plane. Based on this, half-space Green's function $[14,15]$ that automatically satisfies the boundary condition of the reflecting plane was introduced into the equivalent source method- (ESM-) based NAH, and this method performed well [16]. But, this method depends on the surface impedance of the reflecting plane and is named dependence (D)-ESM.

To develop a method for the situations that the surface impedance is unknown and to avoid high measurement costs, the reflections due to the reflecting plane can be regarded as being radiated by equivalent sources located under the plane and then both the direct sound and reflected sound can be expressed as the superposition of a series of equivalent sources [16]. Because this method is based on ESM and is independent of the surface impedance, it is named independent (I)-ESM. It is predictable that I-ESM may give bad reconstruction results and even fail in reconstructing if the equivalent sources are arranged 
improperly because the equivalent source configuration affects ESM heavily.

In [16], two types of configurations of equivalent sources, inside the image source (IIS) and close to the reflecting plane (CRP), are considered to substituting for the reflections in I-ESM. If IIS configuration is used, the reflections are regarded as being radiated by a source with the same geometry of the vibrating object, and this source and the vibrating object are placed symmetrically with respect to the reflecting plane. If CRP configuration is used, the reflections are regarded as being radiated by a planar source coincident with the reflecting plane. The results show that the I-ESM with IIS configuration performs well, whereas the I-ESM with CRP configuration cannot provide stable reconstruction accuracy. This is probably because the equivalent sources substituting for the reflections are set improperly when using CRP configuration, as predicated above.

This paper deals with the optimization of the equivalent source configuration to ensure I-ESM always performs well. Because the problem focuses on the reflected sound, the configuration of equivalent sources substituting for the direct sound is not considered here. The configuration mentioned in the following just considers the equivalent sources substituting for the reflections.

This paper realizes the optimization of equivalent source configuration for I-ESM as follows. Firstly, analyze and discuss the influence of CRP configurations on the reconstruction accuracy to search for the appropriate CRP configurations. Then, study the relation between the appropriate CRP configuration and the IIS configuration and acquire the optimal configurations. Finally, confirm the optimal equivalent source configurations.

\section{Outline of Theory}

According to the idea of I-ESM [16], the sound field above the reflecting plane can be approximated by the superposition of the fields generated by the equivalent sources on a fictitious surface $\Gamma$ within the vibrating object and those on a fictitious surface $\Omega$ substituting for the reflections, as shown in Figure 1. Given a field point $\mathbf{r}$, the pressure can be expressed as

$$
p(r)=i \rho \omega\left[\sum q_{i}^{\Gamma} g_{p}\left(r, r_{i}^{\Gamma}\right)+\sum q_{i}^{\Omega} g_{p}\left(r, r_{i}^{\Omega}\right)\right],
$$

where $i$ means the imaginary, $\rho$ is the density of air, $\omega$ is the angular frequency, $q_{i}^{\Gamma}$ and $q_{j}^{\Omega}$ are the strength of the $i$ th and $j$ th equivalent source on $\Gamma$ and $\Omega$, respectively, $\mathbf{r}_{i}^{\Gamma}$ and $\mathbf{r}_{j}^{\Omega}$ are the position of the $i$ th and $j$ th equivalent source, respectively, and Green's function can be expressed as

$$
\begin{gathered}
g_{p}\left(\mathbf{r}, \mathbf{r}_{i}^{\Gamma}\right)=\frac{e^{i k R_{i}^{\Gamma}}}{4 \pi R_{i}^{\Gamma}}, \\
g_{p}\left(\mathbf{r}, \mathbf{r}_{j}^{\Omega}\right)=\frac{e^{i k R_{j}^{\Omega}}}{4 \pi R_{j}^{\Omega}},
\end{gathered}
$$

where $k$ is the wave number, $R_{i}^{\Gamma}$ is the distance between $\mathbf{r}$ and $\mathbf{r}_{i}^{\Gamma}$, and $R_{j}^{\Omega}$ is the distance between $\mathbf{r}$ and $\mathbf{r}_{j}^{\Omega}$.
Given a hologram surface $H$, equation (1) can be written in a matrix form as

$$
\mathbf{P}^{H}=\mathrm{i} \rho \omega\left(\mathbf{G}_{p}^{\Gamma H} \mathbf{Q}^{\Gamma}+\mathbf{G}_{p}^{\Omega H} \mathbf{Q}^{\Omega}\right)=\mathrm{i} \rho \omega \mathbf{G}_{p}^{\Sigma H} \mathbf{Q}^{\Sigma},
$$

with

$$
\begin{aligned}
\mathbf{G}_{p}^{\Sigma H} & =\left[\begin{array}{ll}
\mathbf{G}_{p}^{\Gamma H} & \mathbf{G}_{p}^{\Omega H}
\end{array}\right], \\
\mathbf{Q}^{\Sigma} & =\left[\begin{array}{ll}
\mathbf{Q}^{\Gamma} & \mathbf{Q}^{\Omega}
\end{array}\right]^{T},
\end{aligned}
$$

where $\mathbf{G}_{p}^{\Gamma H} \mathbf{G}_{p}^{\Omega H}$ are the transfer matrix relating the pressures on $H$ to the equivalent sources on $\Gamma$ and $\Omega$, respectively, and $\mathbf{Q}^{\Gamma}$ and $\mathbf{Q}^{\Omega}$ are the equivalent source strength vector on $\Gamma$ and $\Omega$, respectively. Subsequently, the regularized solution of $\mathbf{Q}^{\Sigma}$ can be obtained as

$$
\mathbf{Q}^{\Sigma}=\frac{1}{\mathrm{i} \rho \omega}\left[\left(\mathbf{G}_{p}^{\Sigma H}\right)^{\mathrm{H}} \mathbf{G}_{p}^{\Sigma H}+\varepsilon \mathbf{E}\right]^{-1}\left(\mathbf{G}_{p}^{\Sigma H}\right)^{\mathrm{H}} \mathbf{P}^{H},
$$

where the superscript " $H$ " denotes the Hermitian transpose, " -1 " denotes the inverse matrix, $\varepsilon$ is the regularization parameter, and $\mathbf{E}$ is a unit matrix.

By substituting $\mathbf{Q}^{\Sigma}$ into equation (3), the pressure at any field point in half-space can be reconstructed. Also, the normal velocity on the surface of the vibrating object can also be reconstructed by using the following formula:

$$
\mathbf{V}^{S}=\mathbf{G}_{v}^{\Sigma S} \mathbf{Q}^{\Sigma}
$$

where $\mathbf{G}_{v}^{\Sigma S}$ is the transfer matrix relating the normal velocities on $S$ to the equivalent sources on $\Gamma$ and $\Omega$, and it can be expressed as

$$
\mathbf{G}_{v}^{\Sigma S}=\left[\begin{array}{ll}
\mathbf{G}_{v}^{\Gamma S} & \mathbf{G}_{v}^{\Omega S}
\end{array}\right],
$$

where $\mathbf{G}_{v}^{\Gamma S}$ and $\mathbf{G}_{v}^{\Omega S}$ are, respectively, constituted of

$$
\begin{aligned}
g_{v}\left(\mathbf{r}_{n}^{S}, \mathbf{r}_{i}^{\Gamma}\right) & =\frac{\left(1-i k R_{j n}^{\Gamma S}\right) e^{i k R_{i n}^{\Gamma S}}}{4 \pi\left(R_{i n}^{\Gamma S}\right)^{2}} \cos \left(\phi_{i n}^{\Gamma S}\right), \\
\cos \left(\phi_{i n}^{\Gamma S}\right) & =\frac{\left(\mathbf{r}_{n}^{S}-\mathbf{r}_{i}^{\Gamma}\right) \cdot \mathbf{n}^{S}}{\left|\mathbf{r}_{n}^{S}-\mathbf{r}_{i}^{\Gamma}\right|}, \\
g_{v}\left(\mathbf{r}_{n}^{S}, \mathbf{r}_{j}^{\Omega}\right) & =\frac{\left(1-i k R_{j n}^{\Omega S}\right) e^{i k R_{j n}^{\Omega S}}}{4 \pi\left(R_{j n}^{\Omega S}\right)^{2}} \cos \left(\phi_{j n}^{\Omega S}\right), \\
\cos \left(\phi_{j n}^{\Omega S}\right) & =\frac{\left(\mathbf{r}_{n}^{S}-\mathbf{r}_{j}^{\Omega}\right) \cdot \mathbf{n}^{S}}{\left|\mathbf{r}_{n}^{S}-\mathbf{r}_{j}^{\Omega}\right|},
\end{aligned}
$$

where "." denotes the dot product operator, $\mathbf{n}^{S}$ is the unit normal vector on the surface of the vibrating object, $R_{i n}^{\Gamma S}$ is the distance between the $n$th surface node $\mathbf{r}_{n}^{S}$ and $\mathbf{r}_{i}^{\Gamma}$, and $R_{j n}^{\Omega S}$ is the distance between $\mathbf{r}_{n}^{S}$ and $\mathbf{r}_{j}^{\Omega}$.

\section{Numerical Simulations}

This section explains optimization of the configuration of equivalent sources substituting for reflections, i.e., the equivalent sources on the fictitious surface $\Omega$, a planar surface coincident with the reflecting plane, as shown in Figure 1. 

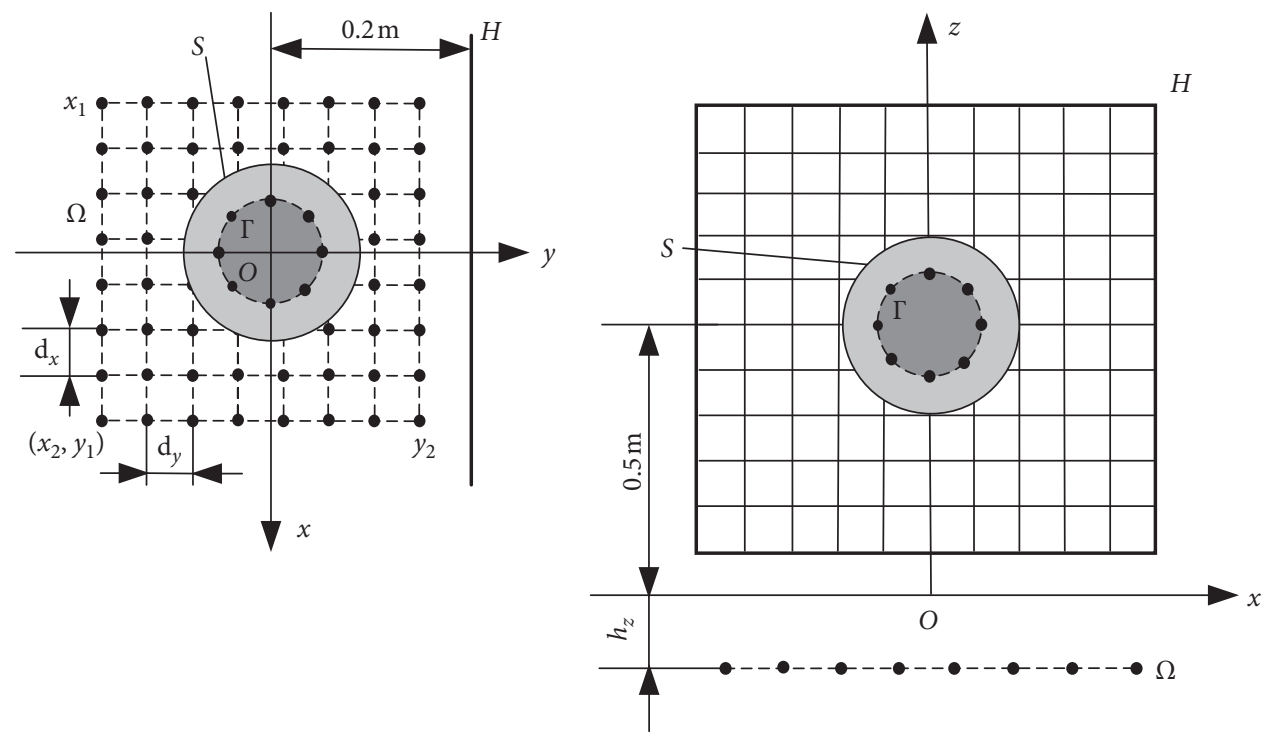

(a)

(b)

Figure 1: Positions of the spherical source $S$, the hologram surface $H$, and the fictitious surface $\Gamma$ and $\Omega$. (a) Top view. (b) Side view.

3.1. Parameters in Numerical Simulations. A spherical source with a radius of $0.1 \mathrm{~m}$ was placed above a reflecting plane. The reflecting plane was assumed to be of the Delany and Bazley type [17] and to be infinitely thick, and then the surface impedance $Z$ was calculated by using the following formula:

$$
Z=\rho c\left[1+9.08\left(\frac{f}{\sigma_{0}}\right)^{-0.75}+i \times 11.9\left(\frac{f}{\sigma_{0}}\right)^{-0.73}\right],
$$

where $f$ is the frequency and $\sigma_{0}$ is the flow resistivity in cgs units. Note that this equation does not take into account the incidence effect; thus, the reflecting plane considered here is of local reaction.

The center of the spherical source was located $0.5 \mathrm{~m}$ above the reflecting plane and the hologram plane $H$ was located at $y=0.2 \mathrm{~m}$, as shown in Figure 1. The dimensions of the hologram plane were $0.5 \mathrm{~m} \times 0.5 \mathrm{~m}$ with measurement points distributed from $-0.25 \mathrm{~m}$ to $0.25 \mathrm{~m}$ in the $x$ direction and from $0.25 \mathrm{~m}$ to $0.75 \mathrm{~m}$ in the $z$ direction, and the sampling interval was $0.05 \mathrm{~m}$ in both $x$ and $z$ directions.

The nodes on the source surface were evenly distributed with intervals of $\pi / 4$ in the azimuth angle direction and $\pi / 6$ in the polar angle direction. The equivalent sources substituting for the direct sound were placed on a smaller concentric spherical surface $\Gamma$ inside the spherical source $S$ with a radius of $0.02 \mathrm{~m}$, which was less than 0.4 times the radius of the spherical source [18]. The distribution of equivalent sources on $\Gamma$ was the same as that of surface nodes.

The normal velocity on the spherical source surface was reconstructed to optimize the equivalent source configuration for I-ESM. The theoretical normal velocity is

$$
v^{S}=v_{0} \frac{z^{S}-z_{a}}{r_{a}}
$$

where $v_{0}$ is the uniform velocity in the radial direction, $r_{a}$ is the radius of the spherical source, and $z^{S}$ and $z_{a}$ are the $z$ coordinates of the source node and the center of the source sphere, respectively. The holographic pressure was calculated numerically by using the boundary element method codes [19]. Also, the surface impedance of the reflecting plane was set as 35 cgs units. Besides, the white noise with a signal-to-noise ratio of $30 \mathrm{~dB}$ was added to the holographic pressure.

3.2. Discussion on the Equivalent Source Configuration. When using CRP configuration, the retreat distance of $\Omega$ from the reflecting plane, $h_{z}$ shown in Figure $1(\mathrm{~b})$, should be considered. Here, $h_{z}$ is set as $-0.001 \mathrm{~m},-0.2 \mathrm{~m},-0.5 \mathrm{~m}$, and $-1 \mathrm{~m}$, respectively. And the dimension of $\Omega$ and the distribution interval of equivalent sources should be considered. The dimension in the $x$ direction is indicated by the minimum and maximum $x$ coordinates, $x_{1}$ and $x_{2}$, as shown in Figure 1(a). Similarly, $y_{1}$ and $y_{2}$ indicate the dimension in the $y$ direction. The distribution intervals are indicated by $d_{x}$ and $d_{y}$. Here, 22 groups of parameters $\left(x_{1}, x_{2}, y_{1}, y_{2}, d_{x}, d_{y}\right)$ are considered and they are given in Table 1 .

With different CRP configurations, the reconstruction errors of normal velocity on the spherical source surface at $500 \mathrm{~Hz}$ were obtained by using I-ESM and they are given in Figure 2.

(1) When $h_{z}$ takes $-0.001 \mathrm{~m},-0.2 \mathrm{~m}$, and $-0.5 \mathrm{~m}$ successively, it is obvious that the reconstruction error decreases with the decrease of $h_{z}$. However, the error does not get smaller when $h_{z}$ takes smaller value $-1 \mathrm{~m}$ and even increases when taking no. 3-12 groups of parameters. Thus, $-0.5 \mathrm{~m}$ is a suitable choice as the retreat distance. It is interesting that the spherical source was located right $0.5 \mathrm{~m}$ above the reflecting plane. So, it can be concluded that the 
TABLe 1: Parameters groups $\left(x_{1}, x_{2}, y_{1}, y_{2}, d_{x}, d_{y}\right)$ in numerical simulations.

\begin{tabular}{lcccc}
\hline Number & $\left(x_{1}, x_{2}\right)(\mathrm{m})$ & $\left(y_{1}, y_{2}\right)(\mathrm{m})$ & $d_{x}(\mathrm{~m})$ & $d_{y}(\mathrm{~m})$ \\
\hline 1 & $(-0.2,0.2)$ & $(-0.2,0.2)$ & 0.050 & 0.050 \\
2 & $(-0.2,0.2)$ & $(-0.2,0.2)$ & 0.025 & 0.025 \\
3 & $(-0.1,0.1)$ & $(-0.1,0.1)$ & 0.050 & 0.050 \\
4 & $(-0.1,0.1)$ & $(-0.1,0.1)$ & 0.025 & 0.025 \\
5 & $(-0.1,0.1)$ & $(-0.1,0.1)$ & 0.010 & 0.010 \\
6 & $(-0.05,0.05)$ & $(-0.05,0.05)$ & 0.050 & 0.050 \\
7 & $(-0.05,0.05)$ & $(-0.05,0.05)$ & 0.025 & 0.025 \\
8 & $(-0.05,0.05)$ & $(-0.05,0.05)$ & 0.010 & 0.010 \\
9 & $(-0.25,0.25)$ & $(0.1,0.2)$ & 0.050 & 0.050 \\
10 & $(-0.25,0.25)$ & $(0.1,0.2)$ & 0.050 & 0.025 \\
11 & $(-0.25,0.25)$ & $(0,0.2)$ & 0.050 & 0.050 \\
12 & $(-0.25,0.25)$ & $(0,0.2)$ & 0.050 & 0.025 \\
13 & $(-0.25,0.25)$ & $(-0.1,0.2)$ & 0.050 & 0.050 \\
14 & $(-0.25,0.25)$ & $(-0.1,0.2)$ & 0.050 & 0.025 \\
15 & $(-0.25,0.25)$ & $(-0.2,0.2)$ & 0.050 & 0.050 \\
16 & $(-0.25,0.25)$ & $(-0.2,0.2)$ & 0.050 & 0.025 \\
17 & $(-0.25,0.25)$ & $(-0.1,0.1)$ & 0.050 & 0.050 \\
18 & $(-0.25,0.25)$ & $(-0.1,0.1)$ & 0.050 & 0.025 \\
19 & $(-0.25,0.25)$ & $(-0.1,0.1)$ & 0.050 & 0.010 \\
20 & $(-0.25,0.25)$ & $(-0.05,0.05)$ & 0.050 & 0.050 \\
21 & $(-0.25,0.25)$ & $(-0.05,0.05)$ & 0.050 & 0.025 \\
22 & $(-0.25,0.25)$ & $(-0.05,0.05)$ & 0.050 & 0.010 \\
\hline
\end{tabular}

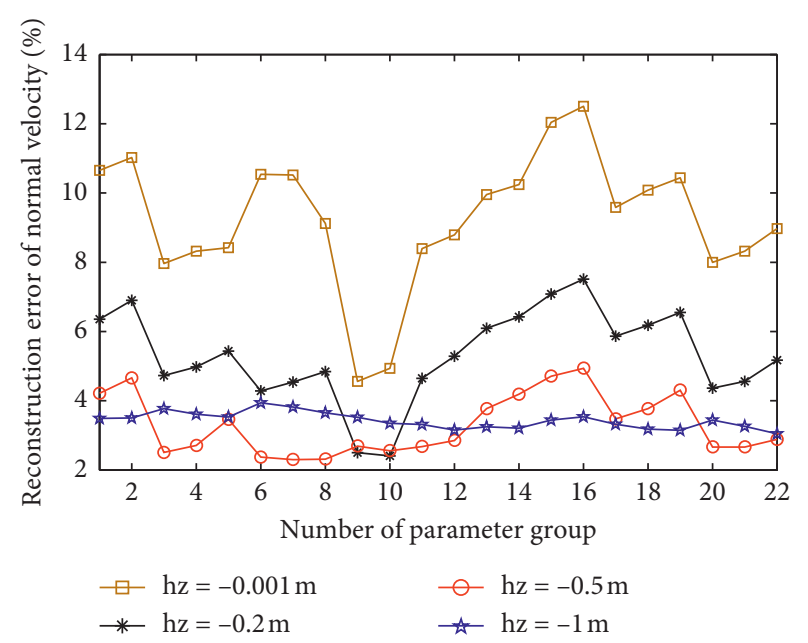

FIgURE 2: The reconstruction errors of normal velocity at $500 \mathrm{~Hz}$ when using different CRP configurations.

retreat distance below the plane should approximate with the distance of the spherical source above the plane, i.e., $\Omega$ should be placed at the distance of the source image.

(2) In the case $h_{z}<-0.5 \mathrm{~m}$, i.e., $h_{z}=-1 \mathrm{~m}, \Omega$ is beyond the distance of the source image. It can be seen that the reconstruction error changes little versus the group of parameters and generally can be acceptable.

(3) In the case $h_{z}>-0.5 \mathrm{~m}$, i.e., $h_{z}=-0.001 \mathrm{~m}$ and $h_{z}=-0.2 \mathrm{~m}, \Omega$ is within the distance of the source image. It can be seen that the two error curves are consistent, showing the same turning points. From Table 1, it can be found that the turning points are just the cases that the dimension $\left(x_{1}, x_{2}, y_{1}, y_{2}\right)$ changes. But, the error changes little with the distribution interval $\left(d_{x}, d_{y}\right)$. This shows that the dimension has heavy influence on the error, while the distribution interval affects the error little.

Besides, it can be seen that the error was the smallest when taking No. 9 group of parameters and was the largest when taking No. 15 group of parameters. Figure 3 gives $\Omega_{9}$ and $\Omega_{15}$, which are, respectively, drawn by No. 9 and No. 15 group of parameters. It can be seen that $\Omega_{9}$ just covers the area between the source and hologram plane and $\Omega_{15}$ is the largest surface covering $\Omega_{9}$. This indicates that the equivalent sources should be arranged just in the area between the source and hologram plane when $\Omega$ is within the distance of the source image.

(4) In the case $h_{z}=-0.5 \mathrm{~m}, \Omega$ is right at the distance of the source image. It can be found that the reconstruction error is quite small when taking No. 3, No. 6, No. 9, No. 11, or No. 20 group of parameters. According to Figure $3, \Omega_{3}$ and $\Omega_{20}$ just cover the spherical source and $\Omega_{6}$ gets smaller covering the central part of the spherical source. Also, $\Omega_{9}$ just covers the area between the source and the hologram plane and $\Omega_{11}$ also covers this area though with a little larger dimension.

In summary, the equivalent sources substituting for the reflections should be placed at either the position of the source image or the area between the source and hologram plane. How interesting it is that the equivalent sources distribute just within the source image when using IIS configuration. Thus, it is expectable that the I-ESM with IIS configuration can perform well.

\subsection{Confirmation of the Optimal Equivalent Source} Configuration. To confirm the optimal equivalent source configuration, Table 2 gives three sets of configurations for further investigation. The first set $\left(h_{z}=-0.001 \mathrm{~m}\right.$, No. 15) represents the inappropriate configuration, while the third set $\left(h_{z}=-0.5 \mathrm{~m}\right.$, No. 6) represents the optimal configuration. Also, the second set $\left(h_{z}=-0.2 \mathrm{~m}\right.$, No. 1$)$ represents an acceptable configuration.

With the configurations given in Table 2, the reconstruction errors of normal velocity were obtained by using I-ESM. Because the D-ESM performs well, it was also used to obtain the reconstruction error, and the error acts as the reference.

Figure 4 gives the reconstruction error of normal velocity versus the frequency, and Figure 5 gives the reconstruction error versus the flow resistivity at $500 \mathrm{~Hz}$. Both figures illustrate that the errors obtained by using I-ESM with $h_{z}=$ $-0.5 \mathrm{~m}$ are comparable to the reference errors, while the errors obtained by using I-ESM with $h_{z}=-0.001 \mathrm{~m}$ are much larger than those. This confirms that the optimal configuration, placing the equivalent sources at the position of source image, is indeed a good choice. 


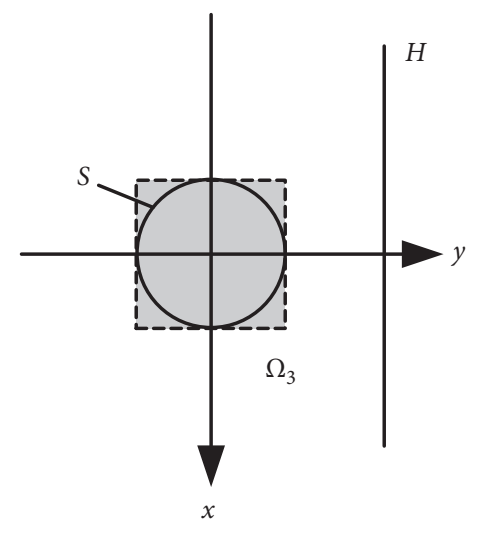

(a)

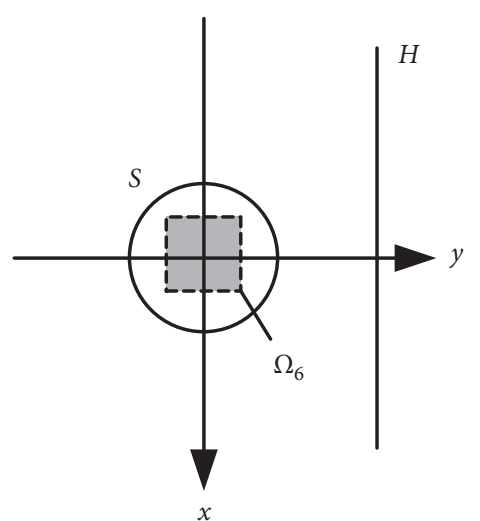

(b)

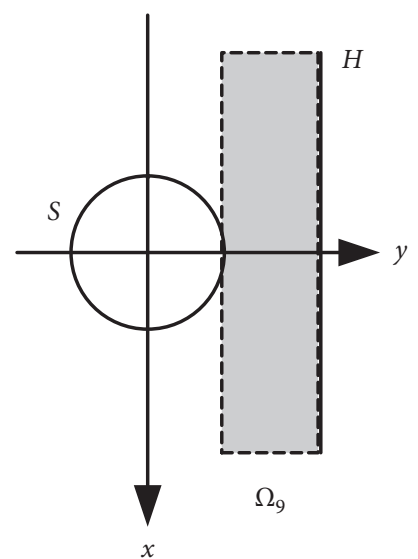

(c)

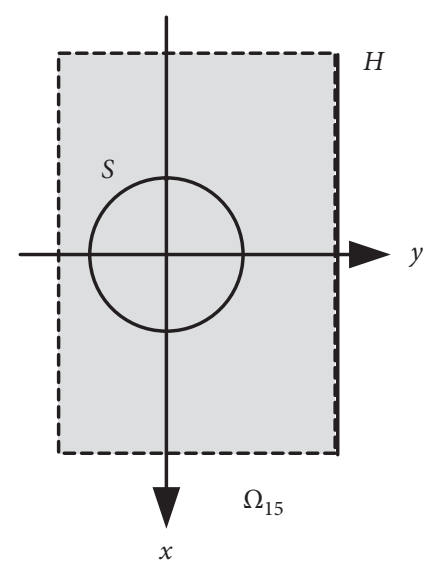

(d)

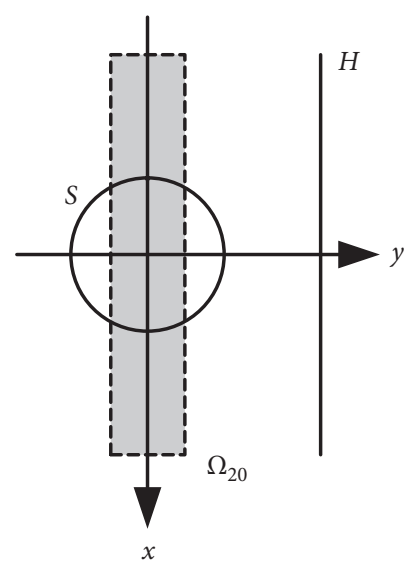

(e)

Figure 3: Geometric sketch of the fictitious surface $\Omega$.

TABLE 2: Equivalent source configurations for confirmation.

\begin{tabular}{lc}
\hline $\begin{array}{l}\text { Retreat distance } \\
h_{z}(\mathrm{~m})\end{array}$ & $\begin{array}{c}\text { Number of parameter group }\left(x_{1}, x_{2}, y_{1}, y_{2},\right. \\
\left.d_{x}, d_{y}\right)\end{array}$ \\
\hline-0.001 & 15 \\
-0.2 & 1 \\
-0.5 & 6 \\
\hline
\end{tabular}

\section{Experiments}

Two experiments were carried out in a semianechoic chamber to optimize the equivalent source configuration for I-ESM. Also, two kinds of reflecting materials, a marble floor and a piece of sponge, were, respectively, used as the reflecting plane.

4.1. Parameters in Experiments. A loudspeaker with the size of $0.60 \mathrm{~m} \times 0.15 \mathrm{~m} \times 0.14 \mathrm{~m}$ was used as the source. Also, the positions of source and measurement surfaces are shown in Figure 6. It can be seen that the loudspeaker was placed $0.18 \mathrm{~m}$ above the reflecting plane and the centers of two cores of the loudspeaker were located at the points $(-0.15 \mathrm{~m}, 0$, $0.18 \mathrm{~m})$ and $(0.10 \mathrm{~m}, 0,0.18 \mathrm{~m})$. The measurement planes $H_{1}$ and $H_{2}$ were located $0.14 \mathrm{~m}$ and $0.20 \mathrm{~m}$ away from the source plane, respectively. The dimensions of the measurement

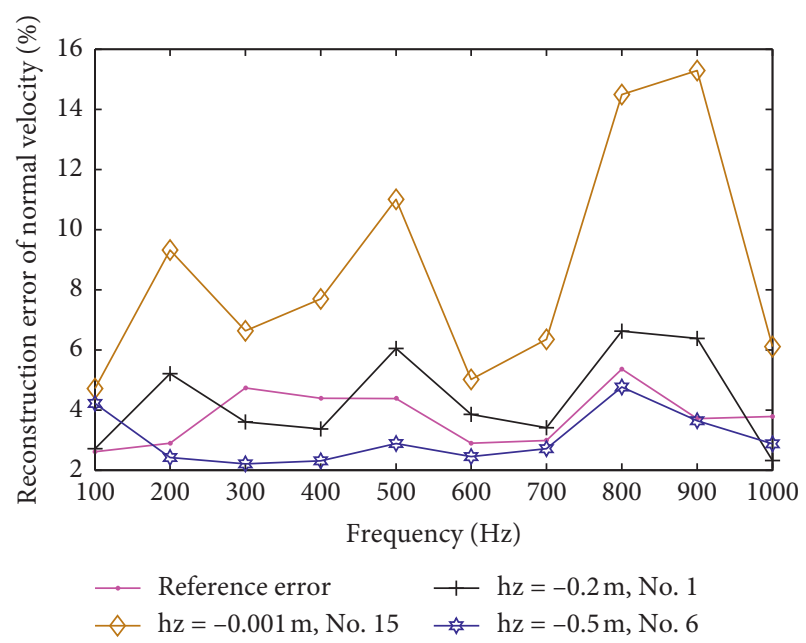

Figure 4: The reconstruction error of normal velocity versus the frequency.

surface were $0.7 \mathrm{~m} \times 0.4 \mathrm{~m}$ with the interval of $0.05 \mathrm{~m}$ in both $x$ and $z$ directions. The fictitious surface $\Gamma$ was located at $y=-0.01 \mathrm{~m}$ with the same dimension and interval as those of measurement surfaces.

Using the measured pressure on $\mathrm{H}_{2}$ as the input, the pressure on $H_{1}$ was reconstructed by using I-ESM, and the 


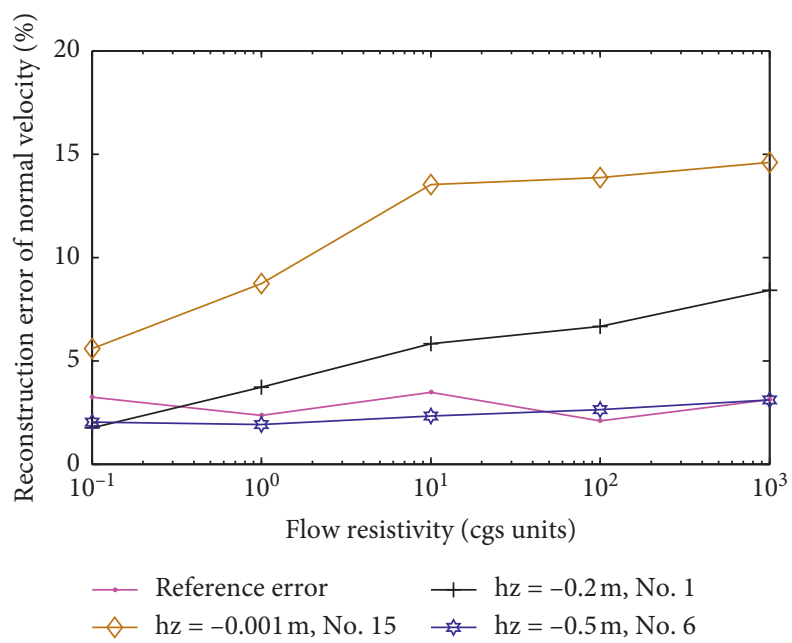

FIGURE 5: The reconstruction error of normal velocity versus the flow resistivity.

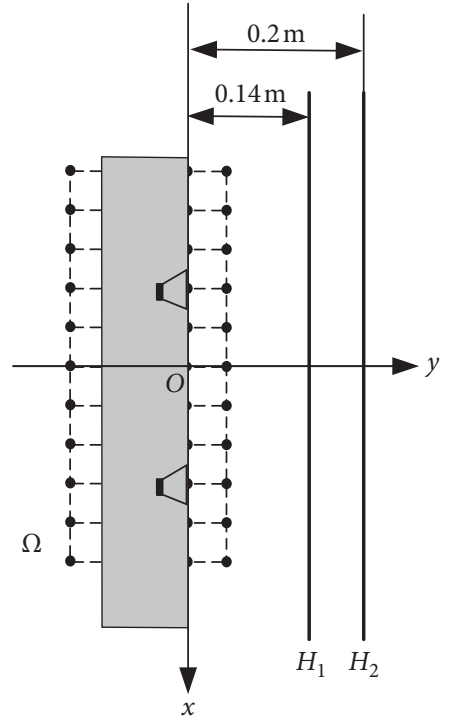

(a)

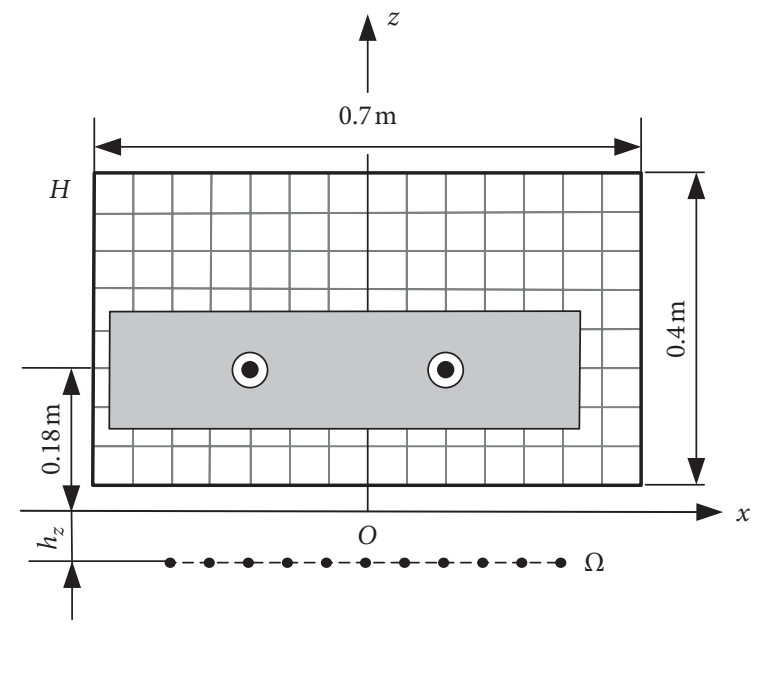

(b)

Figure 6: Location of the loudspeaker, the measurement surfaces $\mathrm{H}_{1}$ and $\mathrm{H}_{2}$, and the fictitious surface $\Omega$. (a) Top view. (b) Side view.

reconstructed results were compared with the measured values on $H_{1}$. Then, the pressure reconstruction error was used to realize the optimization of equivalent source configuration for I-ESM.

\subsection{Discussion on the Equivalent Source Configuration.} Table 3 gives 23 groups of parameters $\left(x_{1}, x_{2}, y_{1}, y_{2}, d_{x}, d_{y}\right)$ to show the dimension and distribution interval of $\Omega$. Also, the retreat distance $h_{z}$ is set as $-0.001 \mathrm{~m},-0.18 \mathrm{~m},-0.5 \mathrm{~m}$ and $-1 \mathrm{~m}$, respectively.

Figure 7 gives the reconstruction errors of pressure at $700 \mathrm{~Hz}$ obtained by using I-ESM with different CRP configurations when a marble floor was used as the reflecting plane.

(1) It is obvious that the reconstruction error is the smallest when $h_{z}=-0.18 \mathrm{~m}$, showing that $-0.18 \mathrm{~m}$ is a nearly perfect choice as the retreat distance. Also, the loudspeaker was placed right $0.18 \mathrm{~m}$ above the reflecting plane. This illustrates that the retreat distance below the reflecting plane should be set as the distance of the source image, which is consistent with the conclusion obtained in numerical simulations.

(2) In the case $h_{z}<-0.18 \mathrm{~m}$, i.e., $h_{z}=-0.5 \mathrm{~m}$ and $h_{z}=-1 \mathrm{~m}$, it can be seen that the reconstruction results are rather stable, but all of them are a little large.

(3) In the case $h_{z}>-0.18 \mathrm{~m}$, i.e., $h_{z}=-0.001 \mathrm{~m}$, it can be seen that the error has large fluctuations versus the group of parameters. Each time the dimension $\left(x_{1}, x_{2}, y_{1}, y_{2}\right)$ changes, the error changes significantly. Also, the distribution interval $\left(d_{x}, d_{y}\right)$ also affects the error much. Thus, if the dimension and 
TABle 3: Parameters groups $\left(x_{1}, x_{2}, y_{1}, y_{2}, d_{x}, d_{y}\right)$ in experiments.

\begin{tabular}{|c|c|c|c|c|}
\hline Number & $\left(x_{1}, x_{2}\right)(\mathrm{m})$ & $\left(y_{1}, y_{2}\right)(\mathrm{m})$ & $d_{x}(\mathrm{~m})$ & $d_{y}(\mathrm{~m})$ \\
\hline 1 & $(-0.2,0.2)$ & $(-0.2,0.2)$ & 0.050 & 0.050 \\
\hline 2 & $(-0.2,0.2)$ & $(-0.2,0.2)$ & 0.025 & 0.025 \\
\hline 3 & $(-0.15,0.15)$ & $(-0.15,0.15)$ & 0.050 & 0.050 \\
\hline 4 & $(-0.15,0.15)$ & $(-0.15,0.15)$ & 0.025 & 0.025 \\
\hline 5 & $(-0.15,0.15)$ & $(-0.15,0.15)$ & 0.010 & 0.010 \\
\hline 6 & $(-0.1,0.1)$ & $(-0.1,0.1)$ & 0.050 & 0.050 \\
\hline 7 & $(-0.1,0.1)$ & $(-0.1,0.1)$ & 0.025 & 0.025 \\
\hline 8 & $(-0.1,0.1)$ & $(-0.1,0.1)$ & 0.010 & 0.010 \\
\hline 9 & $(-0.35,0.35)$ & $(0,0.2)$ & 0.050 & 0.050 \\
\hline 10 & $(-0.35,0.35)$ & $(0,0.2)$ & 0.050 & 0.025 \\
\hline 11 & $(-0.35,0.35)$ & $(-0.15,0.2)$ & 0.050 & 0.050 \\
\hline 12 & $(-0.35,0.35)$ & $(-0.15,0.2)$ & 0.050 & 0.025 \\
\hline 13 & $(-0.35,0.35)$ & $(-0.2,0.2)$ & 0.050 & 0.050 \\
\hline 14 & $(-0.35,0.35)$ & $(-0.2,0.2)$ & 0.050 & 0.025 \\
\hline 15 & $(-0.35,0.35)$ & $(0,0.15)$ & 0.050 & 0.050 \\
\hline 16 & $(-0.35,0.35)$ & $(0,0.15)$ & 0.050 & 0.025 \\
\hline 17 & $(-0.35,0.35)$ & $(0,0.15)$ & 0.050 & 0.010 \\
\hline 18 & $(-0.35,0.35)$ & $(-0.15,0.15)$ & 0.050 & 0.050 \\
\hline 19 & $(-0.35,0.35)$ & $(-0.15,0.15)$ & 0.050 & 0.025 \\
\hline 20 & $(-0.35,0.35)$ & $(-0.15,0.15)$ & 0.050 & 0.010 \\
\hline 21 & $(-0.35,0.35)$ & $(-0.1,0.1)$ & 0.050 & 0.050 \\
\hline 22 & $(-0.35,0.35)$ & $(-0.1,0.1)$ & 0.050 & 0.025 \\
\hline 23 & $(-0.35,0.35)$ & $(-0.1,0.1)$ & 0.050 & 0.010 \\
\hline
\end{tabular}

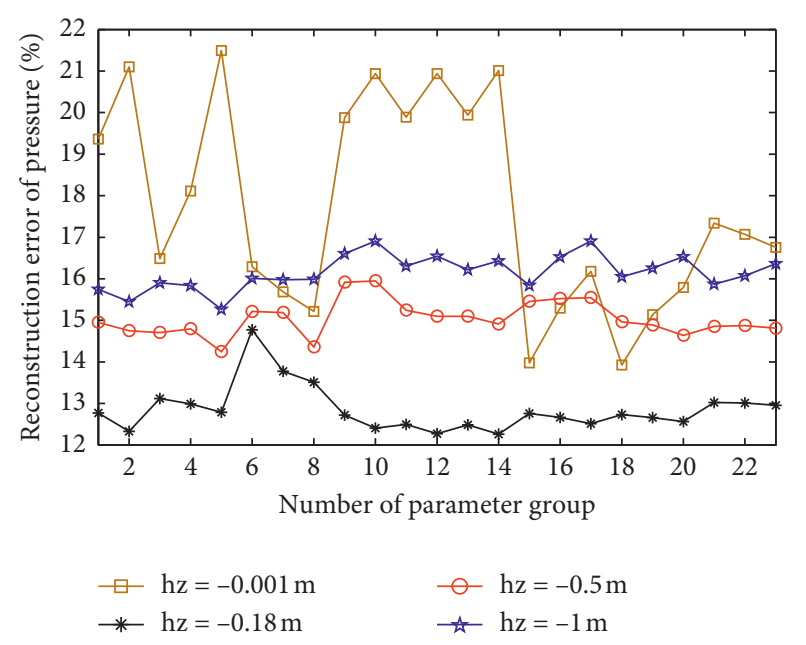

FIgURE 7: The reconstruction errors of pressure when using different CRP configurations with a marble floor as the reflecting plane.

distribution interval are set properly, e.g. taking No. 15 or No. 18 group of parameters, the error can be quite small. From Table 3 , it can be found that $\Omega_{15}$ just covers the area between the source and the reconstructed plane and $\Omega_{18}$ also covers this area though with a little larger dimensions.

(4) In the case $h_{z}=-0.18 \mathrm{~m}$, it is obvious that there is an abnormal point, i.e., when taking No. 6 group of parameters. From Table 3 , it can be seen that $\Omega_{6}$ does not cover both cores of the loudspeaker. Thus, the abnormal point is understandable.
Figure 8 gives the reconstruction errors of pressure at $700 \mathrm{~Hz}$ when a piece of sponge was used as the reflecting plane.

(1) It is obvious that the reconstruction error fluctuates slightly versus the group of parameters when $h_{z} \leq-0.18 \mathrm{~m}$.

(2) In the case $h_{z}>-0.18 \mathrm{~m}$, i.e., $h_{z}=-0.001 \mathrm{~m}$, it can be seen that the error fluctuates significantly versus the group of parameters. Also, the error was the smallest when taking No. 6 or No. 21 group of parameters and the largest when taking No. 13 or No. 14 group of parameters. From Table 3, it can be found that both $\Omega_{6}$ and $\Omega_{21}$ are very small, just covering the central part of the source, while $\Omega_{13}$ and $\Omega_{14}$ are the largest dimensions. In addition, it can be found that the fewer the equivalent sources, the better the reconstruction results. This is probably because that the highly absorptive sponge contributes little for the half-space sound field and few equivalent sources are needed.

(3) In the case $h_{z}=-0.18 \mathrm{~m}$, it can be found that the error is the smallest when taking No. 6 or No. 21 group of parameters. This is consistent with the conclusion presented above that $\Omega$ should just cover the central part of the source and the equivalent sources should be as few as possible.

4.3. Confirmation of the Optimal Equivalent Source Configuration. According to the discussion presented in Section 4.2, Table 4 gives six sets of equivalent source configurations for further investigations to confirm the 


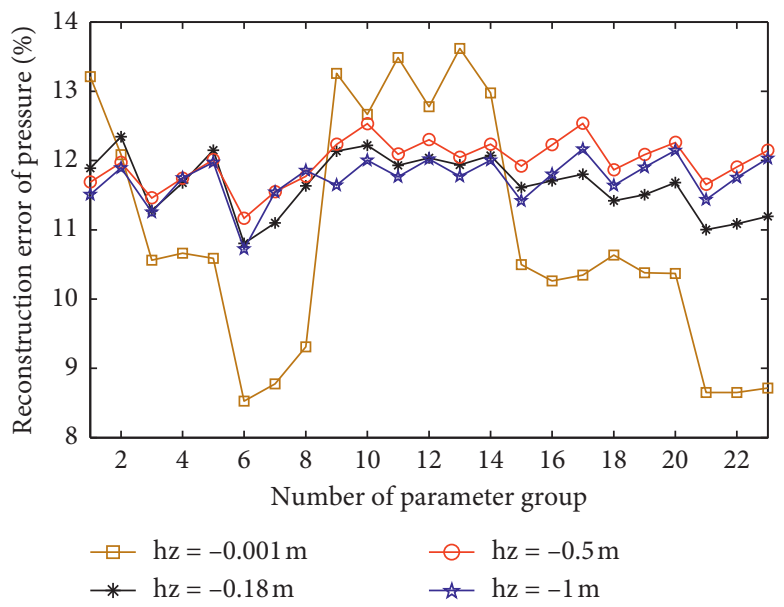

FIGURE 8: The reconstruction errors of pressure when using different CRP configurations with a piece of sponge as the reflecting plane.

TABLE 4: Equivalent source configurations for confirmation in experiments.

\begin{tabular}{lcc}
\hline Reflecting plane & Retreat distance $h_{z}(\mathrm{~m})$ & Number of parameter group $\left(x_{1}, x_{2}, y_{1}, y_{2}, d_{x}, d_{y}\right)$ \\
\hline A marble floor & -0.001 & 5 \\
A marble floor & -0.001 & 18 \\
A marble floor & -0.18 & 6 \\
A piece of sponge & -0.001 & 13 \\
A piece of sponge & -0.001 & 6 \\
A piece of sponge & -0.18 & 21 \\
\hline
\end{tabular}

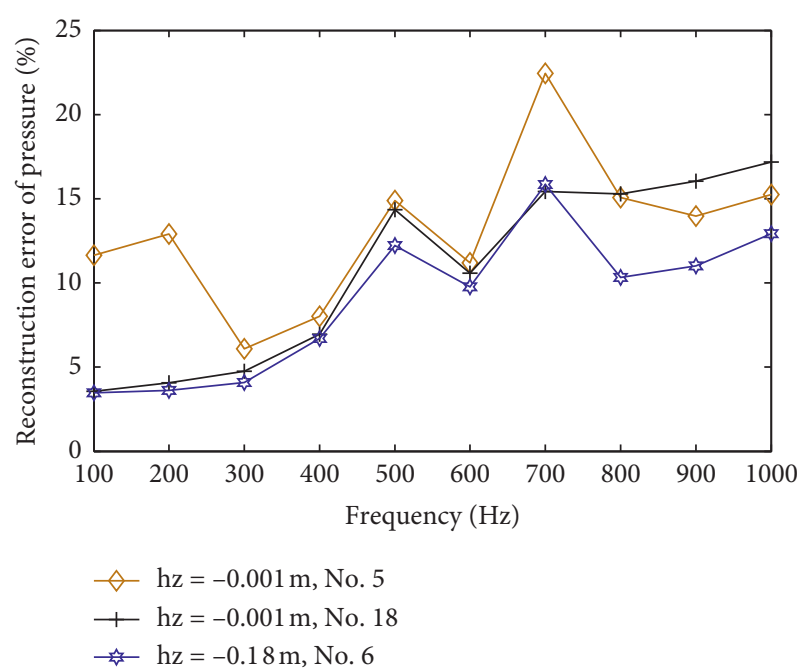

FIGURE 9: The reconstruction error of pressure versus the frequency with a marble floor as the reflecting plane.

optimal configurations. The two configurations when $h_{z}=$ $-0.18 \mathrm{~m}$ represent the optimal ones. Also, other four configurations, respectively, represent the worst and the best in the case $h_{z}=-0.001 \mathrm{~m}$.

Figures 9 and 10 give the reconstruction errors of pressures versus the frequency obtained by using I-ESM with the CRP configurations shown in Table 4 . It can be seen that I-ESM provides large and unstable errors when $h_{z}=-0.001 \mathrm{~m}$ with No. 5 or No. 13 group of parameters. But, the errors obtained when $h_{z}=-0.18 \mathrm{~m}$ are smaller and

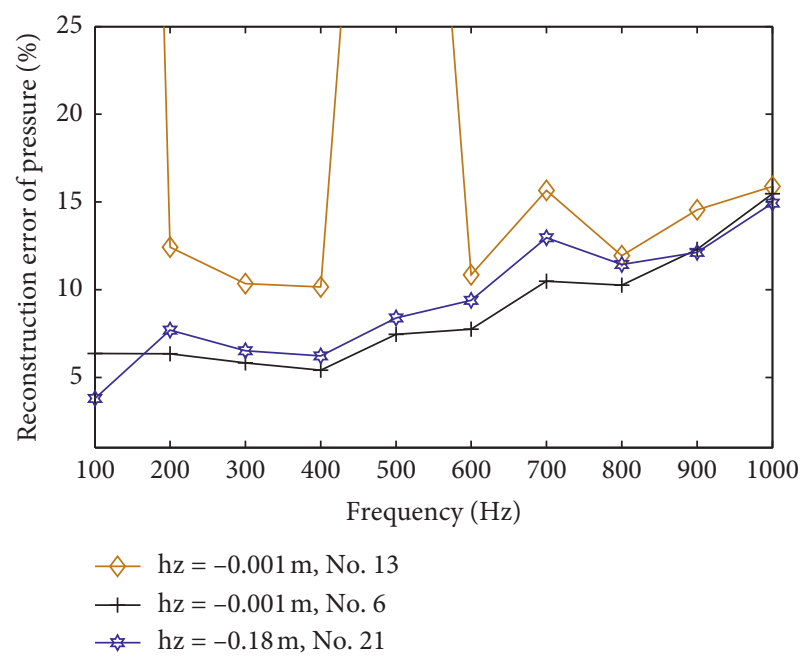

FIGURE 10: The reconstruction error of pressure versus the frequency with a piece of sponge as the reflecting plane.

more stable, confirming that the distance of the source image is suitable to be set as the retreat distance.

Note that the I-ESM performs best when taking No. 6 group of parameters but fails to reconstruct the half-space sound field at $100 \mathrm{~Hz}$ and $500 \mathrm{~Hz}$ when taking No. 13 group of parameters in the case that a piece of sponge was used as the reflecting plane and $h_{z}=-0.001 \mathrm{~m}$. This confirms that placing a small amount of equivalent sources very close to the reflecting plane and just in the central part of source is a good choice when the reflecting plane is highly absorptive. 


\section{Conclusions}

This paper optimizes the configuration of equivalent sources substituting for the reflections for I-ESM. A spherical source was used to carry out numerical simulations, and a loudspeaker was used to carry out two experiments, in which a marble floor and a piece of sponge were, respectively, used as the reflecting plane. By analyzing and discussing the influence of the equivalent source configurations on the reconstruction accuracy, the appropriate CRP configuration is acquired. That is, the equivalent sources should be placed at the position of the image source, verifying that the IIS configuration is indeed an appropriate one. But, if the reflecting plane is consisted of highly absorptive material, such as sponge, it will be better to place few equivalent sources very closely to the plane and just at the central part of source projection.

Moreover, both the numerical simulations and experiments indicate that the optimal equivalent source configuration can improve the performance of I-ESM.

\section{Data Availability}

The data used to support the findings of this study are available from the corresponding author upon request.

\section{Conflicts of Interest}

The authors declare that they have no conflicts of interest.

\section{Acknowledgments}

This work was supported by the National Natural Science Foundation of China (Grant no. 11704110), Major Fund Project of Technical Innovation in Hubei (2017AAA133), and Hubei Superior and Distinctive Discipline Group of "Mechatronics and Automobiles" (XKQ2018002).

\section{References}

[1] E. G. Williams and J. D. Maynard, "Holographic imaging without the wavelength resolution limit," Physical Review Letters, vol. 45, no. 7, pp. 554-557, 1980.

[2] E. G. Williams, Fourier Acoustics: Sound Radiation and Nearfield Acoustical Holography, Academic Press, San Diego, CL, USA, 1999.

[3] L. Geng, L. Yu, M.-L. Mu, C.-D. He, and B. Yan, "Recovery of non-stationary free field based on pressure and acceleration measurements in a noisy environment," Journal of Sound and Vibration, vol. 453, pp. 25-40, 2019.

[4] S. Y. Zhao, Z. G. Chu, Y. Yang, and Y. X. Zhang, "Highresolution CLEAN-SC for acoustic source identification with spherical microphone arrays," Journal of the Acoustical Society of America, vol. 145, pp. 598-603, 2019.

[5] D.-Y. Hu, H.-B. Li, Y. Hu, and Y. Fang, "Sound field reconstruction with sparse sampling and the equivalent source method," Mechanical Systems and Signal Processing, vol. 108, pp. 317-325, 2018.

[6] C.-X. Bi, W.-Q. Jing, and Y.-B. Zhang, "Broadband acoustic holography from intensity measurements with a three-dimensional pressure-velocity probe," The Journal of the
Acoustical Society of America, vol. 138, no. 5, pp. 2929-2936, 2015.

[7] C. Langrenne, A. G. Melon, M. Melon, and A. Garcia, "Boundary element method for the acoustic characterization of a machine in bounded noisy environment," The Journal of the Acoustical Society of America, vol. 121, no. 5, pp. 27502757, 2007.

[8] E. Fernandez-Grande and F. Jacobsen, "Sound field separation with a double layer velocity transducer array (L)," The Journal of the Acoustical Society of America, vol. 130, no. 1, pp. 5-8, 2011.

[9] X. Zhao and S. F. Wu, "Reconstruction of vibroacoustic fields in half-space by using hybrid near-field acoustical holography," The Journal of the Acoustical Society of America, vol. 117, no. 2, pp. 555-565, 2005.

[10] C. Bi, X. Z. Chen, J. Chen, and R. Zhou, "Nearfield acoustic holography based on the equivalent source method," Science in China Series E, vol. 48, no. 3, pp. 338-353, 2005.

[11] C. X. Bi, X. Z. Chen, R. Zhou, and J. Chen, "Reconstruction and separation in a semi-free field by using the distributed source boundary point method-based nearfield acoustic holography," Journal of Vibration and Acoustics, vol. 129, no. 3, pp. 323-329, 2007.

[12] S. Pan, W. Jiang, H. Zhang, and S. Xiang, "Modeling transient sound propagation over an absorbing plane by a half-space interpolated time-domain equivalent source method," The Journal of the Acoustical Society of America, vol. 136, no. 4, pp. 1744-1755, 2014.

[13] S. Pan, W. Jiang, S. Xiang, and X. Liu, "An interpolated timedomain equivalent source method for modeling transient acoustic radiation over a mass-like plane based on the transient half-space Green's function," Wave Motion, vol. 51, no. 8, pp. 1273-1287, 2014.

[14] X. Di and K. E. Gilbert, "An exact Laplace transform formulation for a point source above a ground surface," The Journal of the Acoustical Society of America, vol. 93, no. 2, pp. 714-720, 1993.

[15] M. Ochmann, "The complex equivalent source method for sound propagation over an impedance plane," The Journal of the Acoustical Society of America, vol. 116, no. 6, pp. 33043311, 2004.

[16] C.-X. Bi, W.-Q. Jing, Y.-B. Zhang, and W.-L. Lin, "Reconstruction of the sound field above a reflecting plane using the equivalent source method," Journal of Sound and Vibration, vol. 386, pp. 149-162, 2017.

[17] M. E. Delany and E. N. Bazley, "Acoustical properties of fibrous absorbent materials," Applied Acoustics, vol. 3, no. 2, pp. 105-116, 1970.

[18] G. H. Koopmann, L. Song, and J. B. Fahnline, "A method for computing acoustic fields based on the principle of wave superposition," The Journal of the Acoustical Society of America, vol. 86, no. 6, pp. 2433-2438, 1989.

[19] S. Marburg, B. Nolte, R. Bernhard, and S. Wang, Computational Acoustics of Noise Propagation in Fluids: Finite and Boundary Element Methods, Springer-Verlag, Berlin, Germany, 2008. 